\title{
Ovarian Serous Surface Papillary Adenocarcinoma
}

National Cancer Institute

\section{Source}

National Cancer Institute. Ovarian Serous Surface Papillary Adenocarcinoma. NCI

Thesaurus. Code C6256.

A serous adenocarcinoma that arises from the ovary and is characterized by the presence of a papillary architectural pattern. 